\title{
The antihypertensive and diuretic effects of amiloride and of its combination with hydrochlorothiazide
}

\begin{abstract}
The hypotensive effect as well as changes in serum electrolytes and uric acid of amiloride (AM) and of its combination with hydrochlorothiazide (HC) were studied. In five hypertensive patients over a five-day period, AM and HC administered together produced more natriuresis than either alone. Potassium, chloride, and bicarbonate concentrations in the serum showed a smaller alteration from normal with the combination than with HC alone. Twelve additional outpatients with moderate hypertension were followed for twelve weeks with the use of randomized Latin squares in double-blind fashion. A blood pressure-lowering effect of AM by itself or when added to HC was not seen. AM had no discernible effect on serum glucose, uric acid, or chloride concentrations.
\end{abstract}

Hermann Kampffmeyer, M.D., and James Conway, M.D., Ph.D. Ann Arbor, Mich. Department of Internal Medicine, University Hospital, The University of Michigan Medical Center

Amiloride (AM), N-amidino-3,5,-diamino-6-chloropyrazinamide hydrochloride, induces natriuretic effects without potassium loss or rise in serum uric acid. ${ }^{1,4}$ In single doses, AM has been shown to be less potent than hydrochlorothiazide (HC); however, when administered daily in approximately maximal dosage, the natriuretic action of AM is sustained for a longer period so that the negative sodium balance equals that of $\mathrm{HC}$ after 5 days of administration. ${ }^{5}$ It seemed desirable therefore to investigate

This work was supported by a National Institutes of Health training grant in investigational clinical pharmacology (5T1 HE-5526-05) and by 5M01 FR-42-07. Dr. Kampffmeyer was a Merck International Fellow in Clinical Pharmacology.

Received for publication Jan. 22, 1968.

Accepted for publication Feb. 29, 1968. the diuretic and hypotensive effect of AM combined with HC. For this purpose, a short balance study was undertaken to measure the magnitude of the sodium excretion when HC and AM were given together; then in an outpatient trial the antihypertensive effect of each drug given separately was compared with the combination.

\section{Methods}

Balance study. Five untreated patients with established hypertension, diastolic blood pressures between 100 and $130 \mathrm{~mm}$. $\mathrm{Hg}$, and no evidence of renal failure, edema, or symptoms of heart failure were studied in the Clinical Research Unit of this hospital. Sodium intake was $95 \mathrm{mEq}$. per day. Drugs were administered after 
3 to 5 days of hospitalization, $\mathrm{HC}$ in a dosage of $50 \mathrm{mg}$. twice a day, and AM $10 \mathrm{mg}$. twice a day. There was no restriction of water or potassium; no cathartics were given for 3 days prior to treatment. The collection of specimens and the analyses for the various parameters were as in our previous studies. ${ }^{5}$

\section{Outpatient study.}

Experimental design. Twelve patients received AM ( $20 \mathrm{mg}$. per day) alone, $\mathrm{HC}$ (100 mg. per day) alone, or a combination of both for a period of 3 weeks. These were randomized, 3 patients per group in four Latin squares. The trial was conducted in double-blind fashion. The manufacturer had randomized and coded the drugs for the trial periods and labeled the bottles with patient numbers and period numbers." It was not necessary to break the code until the trial had been completed. The patients took one capsule and one tablet twice daily throughout the whole trial. Prior to receiving active drugs, each patient was given identically appearing placebos for 3 weeks. During this period, investigators (but not the patients) knew that the patients were receiving placebo.

Patients ranging from 34 to 68 years in age already under treatment with $\mathrm{HC}$ for moderate established essential hypertension were chosen. In none was there evidence of malignant hypertension or heart failure; blood pressure had not exceeded $200 / 130 \mathrm{~mm}$. $\mathrm{Hg}$. They had been trained by the clinic nurse to take their blood pressure at home and had reported reliable data for many months. They recorded body weight daily on rising. After an outline of this study had been discussed and an agreement had been obtained from the patient, the subjects received an information sheet explaining the trial in detail.

The trial. The trial took 12 weeks. At

- Supplied by Dr. W. Wilkinson of Merck Sharp \& Dohme, West Point, Pa. the end of each trial period, a 24 hour urine sample was collected for creatinine and sodium determination. On the day of each visit in the outpatient department, a blood sample was drawn for analyses of serum $\mathrm{Na}, \mathrm{K}, \mathrm{Cl}$, creatinine, blood urea nitrogen, glucose, and uric acid concentrations, as well as for hematological and liver function. The remaining tablets from the previous trial period were counted to establish whether the prescribed number had been taken, and the medication for the next period was given. The patients were provided with special forms on which they recorded morning blood pressure readings in recumbent and upright positions and body weights. To check the accuracy of the patients' readings, the nurse as well as the patient measured the blood pressure at each clinic visit.

Analysis of the data. The data for the treatment period have been analyzed by the analysis of variance for a group of Latin squares with a single value in each box. ${ }^{3}$ The two degrees of freedom (d.f.) for treatments were broken down for orthogonal comparison ${ }^{6}$ : 1 d.f. for the comparison between $A M$ and the other two treatments, and 1 d.f. for $\mathrm{HC}$ against the combination of AM and HC. For the test of the $\mathrm{H}_{0}$ hypothesis on the home blood pressure, the mean values of the lying blood pressure in the morning over the last 5 days of each period were entered into each box.

The means obtained with the three different treatments were compared with one another and with placebo with the use of Duncan's Multiple Range Test with the standard error of the pooled variance of the four means. ${ }^{2}$ Obviously, the placebo period could not be included in the analysis of variance.

\section{Results}

Balance study. The results are listed in Table I. The values indicate the means on 5 patients and the corresponding standard errors. On combined therapy over the 5day period, the natriuretic effect was 


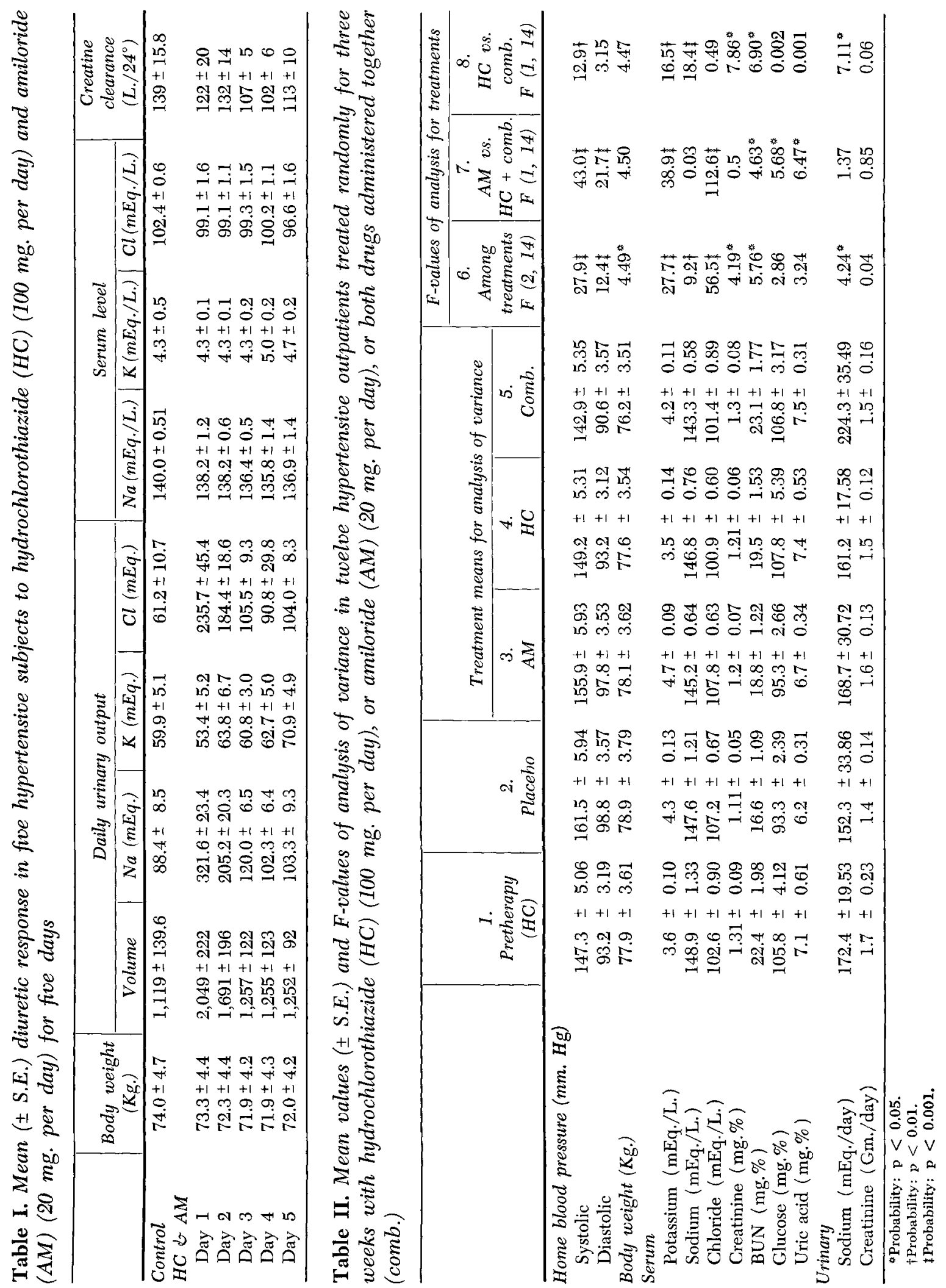


greater than that with either drug alone, ${ }^{5}$ but after 3 days it was only slightly in excess of the dietary intake. The cumulative negative sodium balance calculated was $378 \mathrm{mEq}$. (S.E. $= \pm 26$ ). The serum potassium levels increased from 4.3 to 5.0 $\mathrm{mEq}$. per liter. Serum sodium fell, and the creatinine clearance also fell. The mean recumbent blood pressure fell from 178/ 120 to $160 / 112 \mathrm{~mm}$. $\mathrm{Hg}$.

Outpatient study. Data for this trial are listed in Table II. The mean change in systolic/diastolic blood pressures from placebo averaged $6 / 1 \mathrm{~mm}$. $\mathrm{Hg}$ with $\mathrm{AM}$, $12 / 6 \mathrm{~mm}$. $\mathrm{Hg}$ with $\mathrm{HC}$, and $19 / 8 \mathrm{~mm}$. $\mathrm{Hg}$ with the combination. With the Multiple Range Test used for comparison of blood pressures, there was a difference at the 5 per cent level between the blood pressure on placebo (column 2) and treatment with the combination of $\mathrm{AM}$ and $\mathrm{HC}$ (column $5)$. The mean body weight of 78.9 kilograms on placebo fell 0.8 kilogram on $\mathrm{AM}, 1.3$ kilograms on $\mathrm{HC}$; and 2.7 kilograms on the combination.

The analysis of variance of blood pressure showed differences among the treatments (column 6). The blood pressurelowering effect of $\mathrm{HC}$ and the combination of $\mathrm{HC}$ and $\mathrm{AM}$ was greater than that of AM alone (column 7). The combination showed greater effect on systolic blood pressure than $\mathrm{HC}$ alone, but there was no significant effect on the diastolic blood pressure. The F-values obtained by orthogonal contrast for the comparison between different treatments (columns 7 and 8 ) did not exceed the critical value on the 5 per cent level. The analysis of variance showed no important differences between individuals or groups in the Latin squares or for consecutive time periods.

After 3 weeks of therapy, serum potassium levels were $4.7 \mathrm{mEq}$. per liter on $A \mathrm{M}$, 3.5 on $\mathrm{HC}$, and $4.2 \mathrm{mEq}$. per liter on the combination; these differences among treatments were significant. The serum sodium concentration was lower on the combination of $\mathrm{HC}$ and $\mathrm{AM}$ compared to $\mathrm{HC}$ alone. AM itself did not affect the serum chloride concentration whereas $\mathrm{HC}$ alone and in combination with AM significantly lowered the chloride concentration.

The serum creatinine and BUN concentrations were greater on the combination than on $\mathrm{HC}$ alone but were well in the normal range.

$\mathrm{HC}$ alone or in combination with $\mathrm{AM}$ had more effect on serum glucose levels than AM alone. A similar trend was observed with the serum uric acid. HC increased the mean uric acid level by 1.2 mg. per cent, whereas no increase was seen after AM. Creatinine excretion was not affected.

Undesirable effects. There were no subjective symptoms due to the drugs. Three weeks after treatment with $\mathrm{AM}$ and $\mathrm{HC}$ in combination, one patient then receiving AM alone had an elevated serum glutamic oxalacetic transaminase (168 units). Fourteen days later it had returned to 46 units. The patient had no subjective symptoms and subsequent values were normal.

\section{Discussion}

The blood pressures with $\mathrm{HC}$ treatment before the trial were practically the same as those with $\mathrm{HC}$ during the trial.

AM alone had little effect on the blood pressure and the additional fall when AM was added to $\mathrm{HC}$ was not clinically significant. Thus AM, although previously shown to have as great an effect on sodium balance as $\mathrm{HC},{ }^{5}$ appears to have insignificant antihypertensive properties. This may be accounted for by the possibility that a substantial part of the sodium lost does not come from the extracellular fluid compartment, but may represent an exchange of sodium for cellular potassium. This was thought to be likely since the change in body weight and hematocrit for a given loss of sodium was smaller with AM than it was with HC. ${ }^{5} \mathrm{AM}$ is therefore of special interest as a diuretic since it may cause the withdrawal of sodium from compartments not usually affected by thiazide diuretics, which in special states of edema might be of practical importance. 


\section{References}

1. Baer, J. E., Mucha, C. M., Spitzer, S. A., and Yee, H. W.: A $\mathrm{K}^{+}$-sparing natriuretic pyrazinamide derivative, Proc. Soc. Exper. Biol. \& Med. 25:197, 1966.

2. Duncan, D. B.: Multiple range and multiple F-tests, Biometrics 11:1-42, 1955.

3. Federer, W. T.: Experimental design, New York, 1955, The Macmillan Company, pp. 148152.

4. Gombos, E. A., Freis, E. D., and Moghadam,
A.: Effect of MK-870 in normal subjects and hypertensive patients, New England J. Med. 275:1215-1220, 1966.

5. Hitzenberger, G., Kampffmeyer, H., and Conway, J.: The diuretic effect of desmethyl-pipazuroyl-guanidine (MK-870) in man, CLIN. Pharmacol. \& Therap. 9:71-79, 1968.

6. Li, J. C. R.: Introduction to statistical inference, Ann Arbor, 1961, J. W. Edwards, Publisher, Inc., pp. 227-241. 